\title{
Reevaluating reactor antineutrino anomalies with updated flux predictions
}

\author{
Jeffrey M. Berryman ${ }^{1,2,3}$ and Patrick Huber ${ }^{1}$ \\ ${ }^{1}$ Center for Neutrino Physics, Department of Physics, Virginia Tech, Blacksburg, Virginia 24061, USA \\ ${ }^{2}$ Department of Physics and Astronomy, University of Kentucky, Lexington, Kentucky 40506, USA \\ ${ }^{3}$ Department of Physics, University of California, Berkeley, California 94720, USA
}

(Received 7 October 2019; revised manuscript received 26 November 2019; published 14 January 2020)

\begin{abstract}
Hints for the existence of a sterile neutrino at nuclear reactors are reexamined using two updated predictions for the fluxes of antineutrinos produced in fissions. These new predictions diverge in their preference for the rate deficit anomaly, relative to previous analyses, but the anomaly in the ratios of measured antineutrino spectra persists. We comment on upcoming experiments and their ability to probe the preferred region of the sterile-neutrino parameter space in the electron neutrino disappearance channel.
\end{abstract}

DOI: 10.1103/PhysRevD.101.015008

\section{INTRODUCTION}

The evidence for the existence of additional neutrino species, which we generically call "sterile neutrinos," can be broadly decomposed into three classes: anomalous $\bar{\nu}_{e}$ disappearance at reactors [1]; anomalous $\nu_{e}$ disappearance at GALLEX [2] and SAGE [3], i.e., the gallium anomaly; and anomalous $\bar{\nu}_{e}$ appearance at LSND [4] and MiniBooNE [5]. These individual pieces, however, do not form a consistent whole. The reactor and gallium anomalies were found to be compatible at only the $9 \%$ level in Ref. [6]. Moreover, the combination of these and the absence of anomalous $\nu_{\mu} / \bar{\nu}_{\mu}$ disappearance is incongruous with the LSND and MiniBooNE appearance anomalies. For recent reviews on the status of light sterile neutrinos, see Refs. [7,8].

It is natural to consider, then, why this picture breaks down. While models of new physics have been proposed to explain these anomalies [9-16], an obvious starting point is to scrutinize each to establish how robust it really is. Regarding the gallium anomaly, the ${ }^{71} \mathrm{Ga}\left(\nu_{e}, e^{-}\right)^{71} \mathrm{Ge}$ cross section has recently been reevaluated in Ref. [17] using an updated shell-model calculation. The preference for a sterile neutrino is weaker than for previous calculations, but the compatibility between the gallium and reactor anomalies is improved to $16 \%$ [17]. On the other hand, the LSND and MiniBooNE anomalies will be extensively probed by the upcoming short-baseline program at Fermilab $[18,19]$. In this paper, we focus on the electron neutrino disappearance channel.

Published by the American Physical Society under the terms of the Creative Commons Attribution 4.0 International license. Further distribution of this work must maintain attribution to the author(s) and the published article's title, journal citation, and DOI. Funded by SCOAP ${ }^{3}$.
We have reevaluated the reactor antineutrino anomaly (RAA) using updated predictions for the reactor antineutrino fluxes. The RAA comprises two parts: a deficit in the observed number of $\bar{\nu}_{e}$ interactions relative to predictions and the existence of features in measured $\bar{\nu}_{e}$ spectral ratios. We address both of these in what follows. Our results are obtained with the publicly available software GLOBES $[20,21]$; the underlying data libraries will be published in an accompanying software paper [22].

\section{UPDATED FLUX PREDICTIONS}

Over the past decade, the Huber-Mueller (HM) $\bar{\nu}_{e}$ flux predictions [23,24] have been the standard fluxes for calculations at reactors. These are derived from measurements of the aggregate $\beta$ spectra from the products of nuclear fissions [25-27]. These $\beta$ spectra are then converted to obtain predictions for the corresponding $\bar{\nu}_{e}$ spectra using virtual $\beta$ branches; see Refs. $[23,24]$ for details. While the physics that enters into this procedure is broadly well understood, we highlight two sources of systematic uncertainty. (1) The accuracy of the underlying data. It cannot be excluded that there may be unaccounted for systematics in the measurements of aggregate $\beta$ spectra that bias the results. (2) The theoretical understanding of the component $\beta$ decays. The conversion procedure assumes that all $\beta$ decays are of allowed type. However, a large fraction - up to $40 \%$ - of all decays are so-called forbidden decays. This introduces large uncertainties related to nuclear structure.

An alternative to the conversion method exists in the $a b$ initio method. Here, one adds the spectra from every accessible $\beta$ branch of every fission fragment, with appropriate weights determined by the cumulative fission fraction of each isotope, to determine the overall $\beta$ spectrum.

However, this technique is similarly reliant on experimental data, both for the strengths of the individual 
$\beta$ branches and for the fission yields, which have historically been lacking. In recent years, the $\beta$ feeding data have been revisited and improved using total absorption gamma spectroscopy (TAGS); see, e.g., Ref. [28]. Furthermore, $a b$ initio calculations are also dependent on a theoretical understanding of forbidden decays.

Two new antineutrino flux predictions have recently appeared in the literature. An updated $a b$ initio calculation was recently published in Ref. [28]. There, it was found that $a b$ initio predictions produce better agreement with the $\bar{\nu}_{e}$ spectrum measured at Daya Bay than the HM predictions. This is due, in large part, to a $\sim 10 \%$ reduction in the flux from ${ }^{235} \mathrm{U}$, a feature that is consistent with previous findings $[29,30]$. The improvement largely stems from the improved $\beta$ spectrum feeding functions. However, these calculations do not go beyond the allowed approximation.

An updated conversion method calculation has been presented in Ref. [31]; we refer to this calculation as "HKSS" in the remainder of this work. The significant improvement in HKSS is that forbidden decays are included via nuclear shell model calculations, allowing the authors to derive the relevant shape factors. The authors find an enhancement of the antineutrino flux at energies above $4 \mathrm{MeV}$ relative to $\mathrm{HM}$, somewhat mitigating the size of the infamous $5 \mathrm{MeV}$ bump [30,32-34] and increasing the predicted antineutrino flux.

We have considered the impact of all three of these flux predictions on the preference of the global reactor antineutrino dataset for a sterile neutrino. An important factor in these analyses is the size of the theoretical uncertainties on the flux predictions. The HKSS flux predictions are published with uncertainties; see the appendix to Ref. [31]. In our calculations, we use the uncertainties from their parametrized results. The ab initio fluxes, however, have no stated uncertainties. In the absence of a more compelling option, we assign the fractional uncertainties on the HM predictions to the $a b$ initio predictions in our analysis. This is an optimistic assignment of uncertainties; we will argue, however, that this does not affect the conclusions of this work.

\section{THE RATE ANOMALY}

We begin with combined analyses of the inverse beta decay (IBD) event rates measured at the short-baseline experiments at Bugey [35,36], Gösgen [37], ILL [38,39], Krasnoyarsk [40-42], Nucifer [43], Savannah River [44], and Rovno [45,46]. Additionally, we analyze Chooz [47], Double Chooz [34], and Palo Verde [48,49] at medium baselines, as well as fuel evolution results from Daya Bay [50] and RENO [51,52]. We highlight the salient features of our analysis here; see Ref. [22] for more details.

Our analysis is constructed using ratios of the IBD rates measured at these experiments relative to the three-neutrino predictions for the three reactor antineutrino flux models mentioned previously. We use GLOBES to calculate the total event rate at each experiment as a function of two sterileneutrino parameters-the effective mixing angle $\sin ^{2} 2 \theta_{e e}$ and the mass-squared splitting $\Delta m_{41}^{2}$. For short-baseline experiments, we use the two-flavor approximation for the survival probability,

$$
P_{e e} \approx 1-\sin ^{2} 2 \theta_{e e} \sin ^{2}\left(\frac{\Delta m_{41}^{2} L}{4 E_{\nu}}\right)
$$

For the medium-baseline experiments, we use the full fourflavor oscillation formalism with the best-fit values for the usual three-neutrino oscillation parameters from Ref. [53]. ${ }^{1}$

The differences between the experimental and predicted ratios are combined into a global $\chi^{2}$ function,

$\chi^{2}=\left(\vec{R}_{\text {exp }}-\vec{R}_{\text {pred }}\right)^{T} \cdot V_{\text {exp }}^{-1} \cdot\left(\vec{R}_{\text {exp }}-\vec{R}_{\text {pred }}\right)+\vec{\xi}^{T} \cdot V_{\text {th }}^{-1} \cdot \vec{\xi}$,

where $\vec{R}_{\text {exp }}$ is the vector of experimental ratios, $\vec{R}_{\text {pred }}=$ $\vec{R}_{\text {pred }}\left(\sin ^{2} 2 \theta_{e e}, \Delta m_{41}^{2}, \vec{\xi}\right)$ is the vector of predicted ratios, and $\vec{\xi}$ is a vector of nuisance parameters describing the normalization uncertainties on the isotopic flux predictions-one each for ${ }^{235} \mathrm{U},{ }^{238} \mathrm{U},{ }^{239} \mathrm{Pu}$, and ${ }^{241} \mathrm{Pu}$. Further, $V_{\text {exp }}$ is the covariance matrix describing experimental uncertainties, including correlations, and $V_{\text {th }}$ is the covariance matrix for $\vec{\xi}$. We minimize over the $\vec{\xi}$ for each point in $\sin ^{2} 2 \theta_{e e}-\Delta m_{41}^{2}$ parameter space.

We cross-check our results with the HM flux model against the rate results in Refs. [1,6,54-57]. We find generally good agreement; the resulting $95 \%$ C.L. curve is shown in orange in Fig. 1. The HM fluxes are then replaced in favor of the ab initio and HKSS fluxes and the analysis is repeated; the resulting 95\% C.L. curves are shown, respectively, in blue and dark cyan in Fig. 1. For context, we show the region preferred by the gallium anomaly at $90 \%$ C.L. [17] in shaded purple.

The updated flux models diverge, relative to the HM fluxes, in their preference for a sterile neutrino. On one hand, the $a b$ initio fluxes indicate a much weaker preference for a sterile neutrino; these fluxes prefer nonzero mixing at $<1 \sigma$. This can be largely attributed to the reduced total flux from ${ }^{235} \mathrm{U}$ fissions relative to the $\mathrm{HM}$ predictions, as mentioned above. Further, recall that assigning the HM uncertainties to the $a b$ initio fluxes underestimates the true theoretical uncertainty. A more realistic error budget would further degrade the preference for a sterile neutrino. On the other hand, the HKSS predictions result in stronger evidence for a sterile neutrino: recalculating the shape factor accounting for forbidden decays results in an increased expected IBD rate, implying

\footnotetext{
${ }^{1}$ We assume that the existence of a sterile neutrino has not caused any of these parameters to be mismeasured.
} 


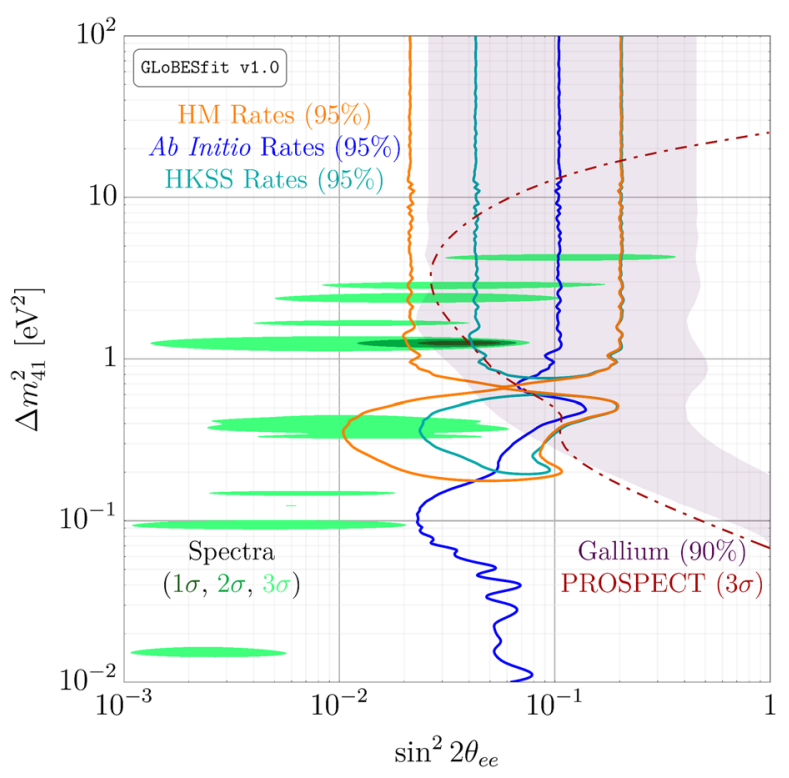

FIG. 1. The 95\% C.L. contours from IBD rate measurements using the HM (orange), ab initio (blue), and HKSS (dark cyan) flux predictions. The regions preferred by reactor antineutrino spectra at $1 \sigma, 2 \sigma$, and $3 \sigma$ are shown in light, medium, and dark green, respectively. We show the $90 \%$ C.L. region preferred by the gallium anomaly [17] for comparison. The red, dot-dashed curve shows the $3 \sigma$ sensitivity of PROSPECT [58] assuming 3 years of operation.

larger experimental deficits. Relevant statistics for these analyses are compiled in Table I.

We underscore that the diverging preference for a sterile neutrino between the $a b$ initio and HKSS flux predictions highlights the need to reappraise the data underpinning these predictions. As of present, improved TAGS measurements in the $a b$ initio model and the more complete treatment of forbidden decays in HKSS modify the total predicted rate to roughly the same degree but with opposite signs. Concerns about vastly increased uncertainties from first-forbidden decays [59] seem not to be borne out in the detailed analysis in HKSS. That said, these conclusions can only be solidified with the collection of more and improved

TABLE I. A summary of relevant statistics in our analyses. We show $\chi^{2}$ for $\sin ^{2} 2 \theta_{e e}=0, \chi_{3 \nu}^{2}$, and the minimum value of $\chi^{2}$ over the sterile neutrino parameter space, $\chi_{\min }^{2}$. We also tabulate the number of data points for each analysis, $n_{\text {data }}$, the $p$-value at which three-neutrino mixing can be excluded and the number of $\sigma$ corresponding to that $p$-value.

\begin{tabular}{lrrrcl}
\hline \hline Analysis & $\chi_{3 \nu}^{2}$ & \multicolumn{1}{c}{$\chi_{\min }^{2}$} & $n_{\text {data }}$ & $p$ & $n \sigma$ \\
\hline HM rates & 41.4 & 33.5 & 40 & $2.0 \times 10^{-2}$ & 2.3 \\
A $b$ initio rates & 39.2 & 37.0 & 40 & 0.34 & 0.95 \\
HKSS rates & 58.1 & 47.5 & 40 & $5.0 \times 10^{-3}$ & 2.8 \\
Spectra & 184.9 & 172.2 & 212 & $1.8 \times 10^{-3}$ & 3.1 \\
DANSS + NEOS & 98.9 & 84.9 & 84 & $9.2 \times 10^{-4}$ & 3.3 \\
DANSS ${ }^{\prime}+$ NEOS & 113.7 & 103.0 & 96 & $4.7 \times 10^{-3}$ & 2.8 \\
\hline \hline
\end{tabular}

data. Until then, this inconclusiveness-that these two new predictions produce diverging evidence relative to $\mathrm{HM}-$ is a primary conclusion of this work.

\section{THE SPECTRAL ANOMALY}

We shift our attention to the reactor $\bar{\nu}_{e}$ energy spectra measured at Bugey [36], DANSS [60], Daya Bay [61], Double Chooz [34], NEOS [32], and RENO [33]. With the exception of NEOS, each of these experiments measures the $\bar{\nu}_{e}$ spectrum at multiple positions and publishes ratios of these spectra. The benefit of such ratios is that the dependence on the reactor flux model largely cancels, mitigating theoretical uncertainties. The NEOS Collaboration presents their spectrum as a ratio with respect to the spectrum measured at Daya Bay in Ref. [62], which introduces mild flux model dependence into the analysis; see Ref. [22] for details.

PROSPECT [63] and STEREO [64,65] have also produced constraints in the last few years. Given that these experiments are still collecting data and that only limited information on how to include them in a global fit is available, we choose not to include them here, but discuss their expected impact below.

The two-flavor approximation in Eq. (1) is used for Bugey, DANSS, and NEOS, but we use the full fourneutrino framework for Daya Bay, Double Chooz, and RENO. These spectral ratios are combined in a single $\chi^{2}$ function of the form

$$
\chi^{2}=\sum_{A}\left(\vec{S}_{\exp }^{A}-\vec{S}_{\text {pred }}^{A}\right)^{T} \cdot\left(V_{A}\right)^{-1} \cdot\left(\vec{S}_{\exp }^{A}-\vec{S}_{\text {pred }}^{A}\right),
$$

where $A$ indexes the experiments, $\vec{S}_{\text {exp }}^{A}$ is the experimental spectral ratio, and $\vec{S}_{\text {pred }}^{A}=\vec{S}_{\text {pred }}^{A}\left(\sin ^{2} 2 \theta_{e e}, \Delta m_{41}^{2}\right)$ is the predicted spectral ratio. Each experiment has its own covariance matrix $V_{A}$ that includes both experimental and theoretical uncertainties. In principle, all experiments are correlated through the theoretical uncertainties. Practically speaking, these correlations are negligible.

The $\chi^{2}$ is calculated at each point in the $\sin ^{2} 2 \theta_{e e}-\Delta m_{41}^{2}$ parameter space; the results are shown in Fig. 1 . The $1 \sigma, 2 \sigma$, and $3 \sigma$ preferred regions are shown in dark, medium, and light green, respectively and are consistent with similar results in Refs. $[6,29,56]$. The sensitivity is primarily driven by DANSS; the total evidence for a sterile neutrino is 3.1 $\sigma$. It is noteworthy that NEOS and DANSS point to the same $\Delta m_{41}^{2}$ despite their baselines differing by a factor of two. Relevant statistics are compiled in Table I.

Recently, the DANSS Collaboration has presented preliminary results for their expanded dataset, including an improved treatment of systematic uncertainties. We have investigated how these data can be expected to modify this analysis. In the last row of Table I, we show relevant statistics for the combination of this new dataset (which we 
call "DANSS'") with NEOS. The overall significance from these data weakens from $3.3 \sigma$ to $2.8 \sigma$; the evidence for a sterile neutrino is lessened, but not altogether washed out. We anticipate revisiting these data upon official publication from the collaboration.

We do not combine our rate and spectral analyses; there are nontrivial correlations that would need to be taken into account that are difficult to estimate. However, one can infer from Fig. 1 that the spectral analysis is consistent with the $a b$ initio analysis; the latter shows weak preference for a sterile neutrino, so consistency is essentially guaranteed. However, one can also infer that the tension between the spectral and HKSS analysis is greater than with the HM analysis. In this way, too, we see the $a b$ initio and HKSS analyses diverge.

It has been suggested that the unexplained feature at $5 \mathrm{MeV}$ in the prompt energy spectrum may influence the inferred evidence for a sterile neutrino. We have performed additional analyses in which additional Gaussian contributions have been added to the HM predictions for ${ }^{235} \mathrm{U}$ and ${ }^{239} \mathrm{Pu}$. These features are taken to have a common central position (5.8 MeV antineutrino energy) and width $(0.5 \mathrm{MeV})$, but they have different magnitudes. These have been determined by comparison with the spectral measurements in Refs. [30,51,66]; see Ref. [22] for more details. We find that the evidence for a sterile neutrino does not meaningfully change in the presence of this bump, for neither rate nor spectral measurements.

\section{FUTURE EXPERIMENTS}

It is useful and imperative to consider how this parameter space can be probed in the near term, given the uncertainty surrounding analyses of the rates but the apparent robustness of spectral measurements. We consider only experiments searching for $\nu_{e} / \bar{\nu}_{e}$ disappearance; for discussions on the future of $\nu_{e} / \bar{\nu}_{e}$ appearance and $\nu_{\mu} / \bar{\nu}_{\mu}$ appearance/ disappearance, see Refs. [7,8].

We begin with PROSPECT and STEREO, which have produced early results [63-65], but not, at present, final analyses. These experiments were designed in the first half of the decade to conclusively probe the RAA as presented in Ref. [1]; early results indicate that they will achieve this. However, since these experiments were conceived, reactor spectrum experiments have shifted the preferred sterile neutrino parameters to smaller mixing angles than previously indicated.

We use PROSPECT as proxy to study how well currentgeneration reactor experiments can probe the regions preferred by the four global analyses presented here. The expected $3 \sigma$ sensitivity for 3 years of operation is shown in dot-dashed dark red in Fig. 1 [58]. This sensitivity represents a prediction of how a null result from PROSPECT, i.e., the nonobservation of oscillations, would constrain the parameter space. The question, then, is how much of the currently allowed regions would survive? To quantify this, we calculate the difference between the threeneutrino $\chi^{2}, \chi_{3 \nu}^{2}$, and the minimum four-neutrino $\chi^{2}$ for fixed $\Delta m_{41}^{2}, \chi_{\min }^{2}\left(\Delta m_{41}^{2}\right)$-a measure of the preference the data show for oscillations with a given $\Delta m_{41}^{2}$-for each analysis presented here. We consider how a null result from PROSPECT would reduce this quantity and hence, how much the allowed parameter space is reduced.

The results are shown in Fig. 2. The dashed curves show $\chi_{3 \nu}^{2}-\chi_{\min }^{2}\left(\Delta m_{41}^{2}\right)$ for the HM rates (orange), ab initio rates (blue), HKSS rates (dark cyan), and spectra (black) analyses with no contribution from PROSPECT. The solid curves are similar, except PROSPECT's sensitivity has been folded in. In regions where the difference between the dashed and solid curves of a given color is large, PROSPECT reduces the allowed parameter space significantly. PROSPECT is effective at probing the HM and HKSS rate analyses in the region $\Delta m_{41}^{2} \sim 1-10 \mathrm{eV}^{2}$; this is precisely the region of parameter space for which it was designed. Conversely, PROSPECT does little to challenge the $a b$ initio rate analysis, since this produces weak preference for a sterile neutrino in the first place.

Most interesting is its sensitivity to the spectral anomaly. Measurements of spectral ratios are susceptible to statistical fluctuations that essentially guarantee a best-fit point at nonzero mixing; see Ref. [67] for more discussion. Consequently, we advocate taking a broader view of the preferred parameter space when considering the impact of PROSPECT on the spectral anomaly. PROSPECT is strongest in probing the region $\Delta m_{41}^{2} \sim 1-5 \mathrm{eV}^{2}$, similar to the HM and HKSS analyses. However, it only has modest power to probe the region $\Delta m_{41}^{2} \sim 0.1-0.5$, where the preference for a sterile neutrino is also nontrivial.



FIG. 2. The difference between the three-neutrino $\chi^{2}, \chi_{3 \nu}^{2}$, and the minimum four-neutrino $\chi^{2}$ for a fixed $\Delta m_{41}^{2}, \chi_{\min }^{2}\left(\Delta m_{41}^{2}\right)$. The dashed lines are for the four global analyses presented here: HM rates (orange), ab initio rates (blue), HKSS rates (dark cyan) and spectra (black); the solid lines show the remaining preference for oscillation after the inclusion of a hypothetical null result from PROSPECT. 
Overall, a $2.4 \sigma$ preference for oscillation would remain even after a null result from PROSPECT. Consequently, additional experimentation would be required to more fully probe the preferred parameter space.

IsoDAR [68], which proposes to use a high-intensity $\bar{\nu}_{e}$ source from $\beta$ decays of ${ }^{8} \mathrm{Li}$, has an expected $5 \sigma$ sensitivity [7] that suggests it has the potential to emphatically confirm or refute the sterile-neutrino interpretation of the RAA. There also exists a burgeoning program of experiments searching for coherent elastic neutrino-nucleus scattering [69-78] that may be able to probe the anomaly $[79,80]$ at high significance, though this process has not yet been observed at a nuclear reactor.

\section{CONCLUSIONS}

We have reanalyzed the global reactor $\bar{\nu}_{e}$ dataset using three reactor antineutrino flux predictions. Relative to the traditional HM predictions, the two new calculations result in diverging evidence for a sterile neutrino when total IBD rate measurements are considered-the ab initio calculation decreases the significance from $2.3 \sigma$ to $<1 \sigma$, whereas the HKSS calculation increases the significance to $2.8 \sigma$. However, the spectral anomaly is robust with respect to varying the flux model and is found to persist at the $3.1 \sigma$ level.
We have shown that null results from current reactor experiments-PROSPECT, in particular-would leave a significant fraction of the currently favored parameter space unexplored. Proclamations of the demise of the light sterile neutrino are, therefore, premature. Given the current evidence for the existence of light sterile neutrinos, it is crucial that the next generation of oscillation experiments includes an effective strategy for probing the sterile neutrino hypothesis in the electron neutrino disappearance channel.

\section{ACKNOWLEDGMENTS}

We thank Giorgio Gratta (Palo Verde), Soo-Bong Kim (RENO), David Lhuillier (STEREO), and Karsten Heeger, and Bryce Littlejohn and Pranava Teja Surukuchi (PROSPECT) for providing data and useful discussions. We also thank Muriel Fallot for providing the $a b$ initio fluxes in machine-readable format and Leendert Hayen for information on the HKSS model. J.M. B. thanks the Fermilab Neutrino Physics Center for their hospitality during the completion of this work. This work is supported by DOE Office of Science Awards No. DE-SC0018327 and No. DE-SC0020262. The work of J. M. B. is also supported by NSF Grant No. PHY-1630782 and by Heising-Simons Foundation Grant No. 2017-228.
[1] G. Mention, M. Fechner, Th. Lasserre, Th. A. Mueller, D. Lhuillier, M. Cribier, and A. Letourneau, The reactor antineutrino anomaly, Phys. Rev. D 83, 073006 (2011).

[2] W. Hampel et al. (GALLEX Collaboration), Final results of the ${ }^{51} \mathrm{Cr}$ neutrino source experiments in GALLEX, Phys. Lett. B 420, 114 (1998).

[3] J. N. Abdurashitov et al. (SAGE Collaboration), Measurement of the response of the Russian-American gallium experiment to neutrinos from a ${ }^{51} \mathrm{Cr}$ source, Phys. Rev. C 59, 2246 (1999).

[4] A. Aguilar-Arevalo et al. (LSND Collaboration), Evidence for neutrino oscillations from the observation of $\bar{\nu}_{e}$ appearance in a $\bar{\nu}_{\mu}$ beam, Phys. Rev. D 64, 112007 (2001).

[5] A. A. Aguilar-Arevalo et al. (MiniBooNE Collaboration), Improved Search for $\bar{\nu}_{\mu} \rightarrow \bar{\nu}_{e}$ Oscillations in the MiniBooNE Experiment, Phys. Rev. Lett. 110, 161801 (2013).

[6] M. Dentler, A. Hernández-Cabezudo, J. Kopp, P. A. N. Machado, M. Maltoni, I. Martinez-Soler, and T. Schwetz, Updated global analysis of neutrino oscillations in the presence of eV-scale sterile neutrinos, J. High Energy Phys. 08 (2018) 010.

[7] A. Diaz, C. A. Argüelles, G. H. Collin, J. M. Conrad, and M. H. Shaevitz, Where are we with light sterile neutrinos? arXiv:1906.00045.
[8] S. Böser, C. Buck, C. Giunti, J. Lesgourgues, L. Ludhova, S. Mertens, A. Schukraft, and M. Wurm, Status of light sterile neutrino searches, arXiv:1906.01739.

[9] A. Merle, S. Morisi, and W. Winter, Common origin of reactor and sterile neutrino mixing, J. High Energy Phys. 07 (2014) 039.

[10] P. Bakhti, Y. Farzan, and T. Schwetz, Revisiting the quantum decoherence scenario as an explanation for the LSND anomaly, J. High Energy Phys. 05 (2015) 007.

[11] K. S. Babu, D. W. McKay, I. Mocioiu, and S. Pakvasa, Light sterile neutrinos, lepton number violating interactions, and the LSND neutrino anomaly, Phys. Rev. D 93, 113019 (2016).

[12] M. Carena, Y.-Y. Li, C. S. Machado, P. A. N. Machado, and C. E. M. Wagner, Neutrinos in large extra dimensions and short-baseline $\nu_{e}$ appearance, Phys. Rev. D 96, 095014 (2017).

[13] G. Magill, R. Plestid, M. Pospelov, and Y.-D. Tsai, Dipole portal to heavy neutral leptons, Phys. Rev. D 98, 115015 (2018).

[14] D. Döring, H. Päs, P. Sicking, and T. J. Weiler, Sterile neutrinos with altered dispersion relations as an explanation for the MiniBooNE, LSND, gallium and reactor anomalies, arXiv:1808.07460. 
[15] J. Liao, D. Marfatia, and K. Whisnant, MiniBooNE, MINOS + and IceCube data imply a baroque neutrino sector, Phys. Rev. D 99, 015016 (2019).

[16] P. B. Denton, Y. Farzan, and I. M. Shoemaker, Activating the fourth neutrino of the $3+1$ scheme, Phys. Rev. D 99, 035003 (2019).

[17] J. Kostensalo, J. Suhonen, C. Giunti, and P. C. Srivastava, The gallium anomaly revisited, Phys. Lett. B 795, 542 (2019).

[18] M. Antonello et al. (MicroBooNE, LAr1-ND, ICARUSWA104 Collaborations), A proposal for a three detector short-baseline neutrino oscillation program in the Fermilab booster neutrino beam, arXiv:1503.01520.

[19] P. A. Machado, O. Palamara, and D. W. Schmitz, The shortbaseline neutrino program at Fermilab, Annu. Rev. Nucl. Part. Sci. 69, 363 (2019).

[20] P. Huber, M. Lindner, and W. Winter, Simulation of longbaseline neutrino oscillation experiments with GLoBES (General Long Baseline Experiment Simulator), Comput. Phys. Commun. 167, 195 (2005).

[21] P. Huber, J. Kopp, M. Lindner, M. Rolinec, and W. Winter, New features in the simulation of neutrino oscillation experiments with GLoBES 3.0: General long baseline experiment simulator, Comput. Phys. Commun. 177, 432 (2007).

[22] J. M. Berryman and P. Huber (to be published).

[23] Th. A. Mueller et al., Improved predictions of reactor antineutrino spectra, Phys. Rev. C 83, 054615 (2011).

[24] P. Huber, On the determination of anti-neutrino spectra from nuclear reactors, Phys. Rev. C 84, 024617 (2011); Erratum, Phys. Rev. C 85, 029901(E) (2012).

[25] F. Von Feilitzsch, A. A. Hahn, and K. Schreckenbach, Experimental beta-spectra from ${ }^{239} \mathrm{Pu}$ and ${ }^{235} \mathrm{U}$ thermal neutron fission products and their correlated antineutrino spectra, Phys. Lett. B 118, 162 (1982).

[26] K. Schreckenbach, G. Colvin, W. Gelletly, and F. Von Feilitzsch, Determination of the antineutrino spectrum from ${ }^{235} \mathrm{U}$ thermal neutron fission products up to $9.5 \mathrm{MeV}$, Phys. Lett. B 160, 325 (1985).

[27] A. A. Hahn, K. Schreckenbach, G. Colvin, B. Krusche, W. Gelletly, and F. Von Feilitzsch, Antineutrino spectra from ${ }^{241} \mathrm{Pu}$ and ${ }^{239} \mathrm{Pu}$ thermal neutron fission products, Phys. Lett. B 218, 365 (1989).

[28] M. Estienne et al., Updated Summation Model: An Improved Agreement with the Daya Bay Antineutrino Fluxes, Phys. Rev. Lett. 123, 022502 (2019).

[29] S. Gariazzo, C. Giunti, M. Laveder, and Y. F. Li, Modelindependent $\bar{\nu}_{e}$ short-baseline oscillations from reactor spectral ratios, Phys. Lett. B 782, 13 (2018).

[30] D. Adey et al. (Daya Bay Collaboration), Measurement of Individual Antineutrino Spectra from ${ }^{235} \mathbf{U}$ and ${ }^{239} \mathbf{P u}$ at Daya Bay, Phys. Rev. Lett. 123, 111801 (2019).

[31] L. Hayen, J. Kostensalo, N. Severijns, and J. Suhonen, Firstforbidden transitions in the reactor anomaly, arXiv:1908 .08302 .

[32] Y. Ko et al. (NEOS Collaboration), Sterile Neutrino Search at the NEOS Experiment, Phys. Rev. Lett. 118, 121802 (2017).

[33] G. Bak et al. (RENO Collaboration), Measurement of Reactor Antineutrino Oscillation Amplitude and Frequency at RENO, Phys. Rev. Lett. 121, 201801 (2018).
[34] H. De Kerret et al. (Double Chooz Collaboration), First double chooz $\theta_{\mathbf{1 3}}$ measurement via total neutron capture detection, arXiv:1901.09445.

[35] Y. Declais et al., Study of reactor anti-neutrino interaction with proton at Bugey nuclear power plant, Phys. Lett. B 338, 383 (1994).

[36] Y. Declais et al., Search for neutrino oscillations at 15-meters, 40-meters, and 95-meters from a nuclear power reactor at Bugey, Nucl. Phys. B434, 503 (1995).

[37] G. Zacek et al. (CALTECH-SIN-TUM Collaboration), Neutrino oscillation experiments at the Gösgen nuclear power reactor, Phys. Rev. D 34, 2621 (1986).

[38] H. Kwon, F. Boehm, A. A. Hahn, H. E. Henrikson, J. L. Vuilleumier, J. F. Cavaignac, D. H. Koang, B. Vignon, F. Von Feilitzsch, and R. L. Mossbauer, Search for neutrino oscillations at a fission reactor, Phys. Rev. D 24, 1097 (1981).

[39] A. Hoummada, S. Lazrak Mikou, M. Avenier, G. Bagieu, J. F. Cavaignac, and Dy. Holm Koan, Neutrino oscillations I.L.L. experiment reanalysis, Appl. Radiat. Isot. 46, 449 (1995).

[40] G. S. Vidyakin, V. N. Vyrodov, I. I. Gurevich, Yu. V. Kozlov, V.P. Martemyanov, S. V. Sukhotin, V. G. Tarasenkov, and S.K. Khakimov, Detection of antineutrinos in the flux from two reactors, Zh. Eksp. Teor. Fiz. 93, 424 (1987) [Sov. Phys. JETP 66, 243 (1987)].

[41] G. S. Vidyakin et al., Limitations on the characteristics of neutrino oscillations, Pis'ma Zh. Eksp. Teor. Fiz. 59, 364 (1994) [JETP Lett. 59, 390 (1994)].

[42] Yu. V. Kozlov, S. V. Khalturtsev, I. N. Machulin, A. V. Martemyanov, V. P. Martemyanov, S. V. Sukhotin, V. G. Tarasenkov, E. V. Turbin, and V. N. Vyrodov, Antineutrinodeuteron experiment at the Krasnoyarsk reactor, Yad. Fiz. 63, 1091 (2000) [Phys. At. Nucl. 63, 1016 (2000)].

[43] G. Boireau et al. (NUCIFER Collaboration), Online monitoring of the Osiris reactor with the Nucifer neutrino detector, Phys. Rev. D 93, 112006 (2016).

[44] Z. D. Greenwood et al., Results of a two position reactor neutrino oscillation experiment, Phys. Rev. D 53, 6054 (1996).

[45] A. I. Afonin, S. N. Ketov, V. I. Kopeikin, L. A. Mikaelyan, M. D. Skorokhvatov, and S. V. Tolokonnikov, A study of the reaction $\bar{\nu}_{e}+P \rightarrow e^{+}+N$ on a nuclear reactor, Zh. Eksp. Teor. Fiz. 94N2, 1 (1988) [Sov. Phys. JETP 67, 213 (1988)].

[46] A. A. Kuvshinnikov, L. A. Mikaelyan, S. V. Nikolaev, M. D. Skorokhvatov, and A. V. Etenko, Measuring the $\bar{\nu}_{e}+p \rightarrow$ $n+e^{+}$cross-section and beta decay axial constant in a new experiment at Rovno NPP reactor (in Russian), JETP Lett. 54, 253 (1991); Sov. J. Nucl. Phys. 52, 300 (1990).

[47] M. Apollonio et al. (CHOOZ Collaboration), Search for neutrino oscillations on a long baseline at the $\mathrm{CHOOZ}$ nuclear power station, Eur. Phys. J. C 27, 331 (2003).

[48] F. Boehm et al., Final results from the Palo Verde neutrino oscillation experiment, Phys. Rev. D 64, 112001 (2001).

[49] G. Gratta (private communication).

[50] F. P. An et al. (Daya Bay Collaboration), Evolution of the Reactor Antineutrino Flux and Spectrum at Daya Bay, Phys. Rev. Lett. 118, 251801 (2017).

[51] G. Bak et al. (RENO Collaboration), Fuel-Composition Dependent Reactor Antineutrino Yield and Spectrum at RENO, Phys. Rev. Lett. 122, 232501 (2019). 
[52] S.-B. Kim (private communication).

[53] I. Esteban, M. C. Gonzalez-Garcia, A. HernándezCabezudo, M. Maltoni, and T. Schwetz, Global analysis of three-flavour neutrino oscillations: Synergies and tensions in the determination of $\theta_{23}, \delta_{C P}$, and the mass ordering, J. High Energy Phys. 01 (2019) 106.

[54] S. Gariazzo, C. Giunti, M. Laveder, and Y. F. Li, Updated global $3+1$ analysis of short-baseline neutrino oscillations, J. High Energy Phys. 06 (2017) 135.

[55] C. Giunti, X. P. Ji, M. Laveder, Y. F. Li, and B. R. Littlejohn, Reactor fuel fraction information on the antineutrino anomaly, J. High Energy Phys. 10 (2017) 143.

[56] M. Dentler, A. Hernández-Cabezudo, J. Kopp, M. Maltoni, and T. Schwetz, Sterile neutrinos or flux uncertainties?Status of the reactor anti-neutrino anomaly, J. High Energy Phys. 11 (2017) 099.

[57] C. Giunti, Y. F. Li, B. R. Littlejohn, and P. T. Surukuchi, Diagnosing the reactor antineutrino anomaly with global antineutrino flux data, Phys. Rev. D 99, 073005 (2019).

[58] J. Ashenfelter et al. (PROSPECT Collaboration), The PROSPECT physics program, J. Phys. G 43, 113001 (2016).

[59] A. C. Hayes, J. L. Friar, G. T. Garvey, G. Jungman, and G. Jonkmans, Systematic Uncertainties in the Analysis of the Reactor Neutrino Anomaly, Phys. Rev. Lett. 112, 202501 (2014).

[60] I. Alekseev et al. (DANSS Collaboration), Search for sterile neutrinos at the DANSS experiment, Phys. Lett. B 787, 56 (2018).

[61] D. Adey et al. (Daya Bay Collaboration), Measurement of the Electron Antineutrino Oscillation with 1958 Days of Operation at Daya Bay, Phys. Rev. Lett. 121, 241805 (2018).

[62] F. P. An et al. (Daya Bay Collaboration), Improved measurement of the reactor antineutrino flux and spectrum at Daya Bay, Chin. Phys. C 41, 013002 (2017).

[63] J. Ashenfelter et al. (PROSPECT Collaboration), First Search for Short-Baseline Neutrino Oscillations at HFIR with PROSPECT, Phys. Rev. Lett. 121, 251802 (2018).

[64] H. Almazán et al. (STEREO Collaboration), Sterile Neutrino Constraints from the STEREO Experiment with 66 Days of Reactor-On Data, Phys. Rev. Lett. 121, 161801 (2018).

[65] L. Bernard (STEREO Collaboration), in 54th Rencontres de Moriond on Electroweak Interactions and Unified Theories (Moriond EW 2019) La Thuile, Italy, 2019 (2019).

[66] J. Ashenfelter et al. (PROSPECT Collaboration), Measurement of the Antineutrino Spectrum from ${ }^{235} \mathrm{U}$ Fission at HFIR with PROSPECT, Phys. Rev. Lett. 122, 251801 (2019).
[67] M. Agostini and B. Neumair, Statistical methods for the search of sterile neutrinos, arXiv:1906.11854.

[68] J. R. Alonso and K. Nakamura (IsoDAR Collaboration), IsoDAR@KamLAND: A conceptual design report for the conventional facilities, arXiv:1710.09325.

[69] H. T. Wong, Ultra-low-energy germanium detector for neutrino-nucleus coherent scattering and dark matter searches, Mod. Phys. Lett. A 23, 1431 (2008).

[70] D. Yu. Akimov et al. (RED Collaboration), Prospects for observation of neutrino-nuclear neutral current coherent scattering with two-phase xenon emission detector, J. Instrum. 8, P10023 (2013).

[71] A. Gútlein et al., Impact of coherent neutrino nucleus scattering on direct dark matter searches based on $\mathrm{CaWO}_{4}$ crystals, Astropart. Phys. 69, 44 (2015).

[72] V. Belov et al., The $\nu$ GeN experiment at the Kalinin Nuclear Power Plant, J. Instrum. 10, P12011 (2015).

[73] A. Aguilar-Arevalo et al. (CONNIE Collaboration), Results of the engineering run of the Coherent Neutrino Nucleus Interaction Experiment (CONNIE), J. Instrum. 11, P07024 (2016).

[74] G. Agnolet et al. (MINER Collaboration), Background studies for the MINER coherent neutrino scattering reactor experiment, Nucl. Instrum. Methods Phys. Res., Sect. A 853, 53 (2017).

[75] J. Billard et al., Coherent neutrino scattering with low temperature bolometers at chooz reactor complex, J. Phys. G 44, 105101 (2017).

[76] R. Strauss et al., The $\nu$-cleus experiment: A gram-scale fiducial-volume cryogenic detector for the first detection of coherent neutrino-nucleus scattering, Eur. Phys. J. C 77, 506 (2017).

[77] D. Akimov et al. (COHERENT Collaboration), Observation of coherent elastic neutrino-nucleus scattering, Science 357, 1123 (2017).

[78] J. Hakenmüller, The CONUS experiment, https://indico .cern.ch/event/606690/contributions/2591545/attachments/ 1499330/2336272/Taup2017_CONUS_talk_JHakenmueller .pdf.

[79] B. C. Cañas, E. A. Garcés, O. G. Miranda, and A. Parada, The reactor antineutrino anomaly and low energy threshold neutrino experiments, Phys. Lett. B 776, 451 (2018).

[80] C. Blanco, D. Hooper, and P. Machado, Constraining sterile neutrino interpretations of the LSND and MiniBooNE anomalies with coherent neutrino scattering experiments, arXiv:1901.08094. 